\title{
Atom Probe Tomography of Interfacial Segregation in CdTe-based Solar Cells
}

Jonathan D. Poplawsky, ${ }^{1}$ Wei Guo, ${ }^{1}$ Naba R. Paudel, ${ }^{2}$ Chen Li, ${ }^{3}$ Amy Ng, ${ }^{4}$ Karren L. More, ${ }^{1}$ Yanfa Yan, ${ }^{2}$ and Stephen J. Pennycook ${ }^{5}$

1. Center for Nanophase Materials Sciences, Oak Ridge National Laboratory, Oak Ridge, TN, USA.

2. Department of Physics and Astronomy, The University of Toledo, Toledo, OH, USA.

3. Department of Lithospheric Research, University of Vienna, Vienna, Austria.

4. Department of Chemistry, Vanderbilt University, Nashville, TN, USA.

5. National University of Singapore, Department of Materials Science and Engineering, Singapore.

Thin film polycrystalline (poly) CdTe-based solar cells are one of the leading commercialized thin-film photovoltaic devices due to their low production costs and high theoretical efficiency $(\sim 33 \%)$. However, there is a large efficiency gap between the highest laboratory-produced thin-film CdTe cell $(21.5 \%)$ and theory [1]. Several processing steps have been developed to increase the device efficiency, including a $\mathrm{CdCl}_{2}$ heat treatment, a $\mathrm{Cu}$ diffusion step, the application of a ZnTe:Cu back contact buffer layer, and the creation of a graded $\mathrm{CdTe}_{\mathrm{x}} \mathrm{Se}_{1-\mathrm{x}}$ layer. [2] Understanding the nanoscale material changes resulting from these processing steps, such as solute segregation to grain boundaries (GBs) and interfaces, is important to further improve the efficiency beyond $25 \%$. Atom probe tomography (APT) can be used to quantify solute segregation to GBs and interfaces [3,4]. The time-of-flight (TOF) mass spectrometry nature of APT makes CdTe materials a challenge to analyze due to the large number of isotopes for Cd and Te, the molecular evaporation preference of CdTe-based materials, and can be especially problematic at the $\mathrm{CdS} / \mathrm{CdTe}$ interface due to several peak overlaps of the $\mathrm{Cl}$ and $\mathrm{S}$ molecular species.

Fig. 1 (a)-(e) shows an APT dataset for the CdS/CdTe interface, and includes the corresponding mass spectra associated with various regions of interest (ROI) within the data. This cell was $\mathrm{CdCl}_{2}$ treated, and thus, quantifying $\mathrm{Cl}$ segregation to $\mathrm{GBs}$ and the interfaces is of interest. $\mathrm{Cl}$ most likely field evaporates as $\mathrm{CdCl}$, but can also evaporate as $\mathrm{CdTeCl}$ or $\mathrm{Te}{ }_{2} \mathrm{Cl}$. The $\mathrm{Cl}$ composition becomes increasingly difficult to quantify close to the CdS/CdTe interface because a $\mathrm{CdTe}_{\mathrm{x}} \mathrm{S}_{1-\mathrm{x}}$ layer with $\sim 5$ at. $\% \mathrm{~S}$ forms several $100 \mathrm{~s}$ of $\mathrm{nm}$ from the $\mathrm{CdS}$ layer due to $\mathrm{S}$ diffusion. $\mathrm{S}$ also field evaporates molecularly as $\mathrm{CdS}, \mathrm{CdTeS}, \mathrm{TeS}$, $\mathrm{S}_{2}, \mathrm{~S}_{3}, \mathrm{Cd}_{2} \mathrm{~S}, \mathrm{Cd}_{2} \mathrm{~S}, \mathrm{Cd}_{2} \mathrm{TeS}, \mathrm{CdTeS}_{2}, \mathrm{Te}_{2} \mathrm{~S}, \mathrm{Cd}_{2} \mathrm{~S}_{2}$, and $\mathrm{Te}_{2} \mathrm{~S}_{2}$ forming several mass spectrum peak overlaps with the $\mathrm{Cl}$ complexes. These peak overlaps only occur within $\mathrm{GBs}$ and interfaces because $\mathrm{Cl}$ does not infiltrate the grain interiors at compositions greater than the detection limit of APT, while S diffuses into the $\mathrm{CdTe}$ grains close to the CdS layer with compositions greater than 5 at. \%. A peak deconvolution routine is employed to accurately quantify the $\mathrm{Cl}$ and $\mathrm{S}$ compositions within interfacial regions. There are also mass spectrum peak overlaps for $\mathrm{Cd}_{2} \mathrm{~S}$, Te, and $\mathrm{Te}_{2}$, making profiles across the CdS/CdTe interface a challenge. These profiles can be normalized to the bulk grain $\mathrm{S}$ and Te compositions by using the IVAS peak deconvolution routine for large volumes with sufficient ion counts to increase the statistical accuracy.

Fig. 1 (f)-(g) shows a STEM electron energy loss spectroscopy (EELS) Cl concentration line-profile and an APT Cl concentration line-profile across a GB. The APT Cl-concentration profile shows a much lower maximum $\mathrm{Cl}$ composition compared to the STEM-EELS data, and the profile encompasses a much larger distance than that of the STEM EELS data. Therefore, a local magnification artifact is revealed within the APT data, which is expected to vary in magnitude with respect to the orientation of each grain boundary. Gaussian functions were fit to ten STEM EELS Cl profiles to obtain an average width in $\mathrm{nm}$ of the $\mathrm{Cl}$ segregation around CdTe GBs. Gaussian functions were also fit to the APT data. The GB 
profile widths for the APT data were normalized to the average STEM-EELS GB width to obtain a corrected maximum $\mathrm{Cl}$ concentration. The maximum $\mathrm{GB} \mathrm{Cl}$ concentration determined by the APT data closely matched that of the STEM-EELS data after this normalization procedure. The Gibbsian excess method can also be used to determine Cl-segregation to GBs analyzed by APT since local magnification artifacts do not affect this measurement, but quantifying Gibbsian excess using STEM-EELS is extraordinarily difficult. Therefore, these results are difficult to compare to STEM EELS measurements. Te-depletion at the CdTe GBs is also observed in the STEM-EELS data (Fig. 1 (f)), which is not observed in the APT data. $\mathrm{Cl}$ substitution for Te is extremely important for understanding the electrical properties of the GBs. The inability for APT to detect Te depletion within CdTe GBs will be discussed.

APT has been used to understand solute segregation to GBs and interfaces in CdTe devices exposed to different treatments, including $\mathrm{CdCl}_{2}, \mathrm{Cu}$ diffusion, and Se diffusion treatments. These results are combined with results from other techniques such as electron beam induced current (EBIC), external quantum efficiency (EQE), and current-voltage (JV) measurements to understand the role of solute segregation in improving the cell efficiencies. Such measurements, and their correlation with fabrication processes and resulting performance, are important for improving CdTe solar cell efficiencies beyond $25 \%$, and can be applied to other solar materials systems. [6]

\section{References:}

[1] M.A. Green et al., Prog. Photovolt: Res. Appl, (2015), 24, p. 3.

[2] N.R. Paudel, Y. Yan, Appl. Phys. Lett, (2014), 105, p. 183510.

[3] P.-P. Choi et al., Micros. Today, (2012), 20, p. 18.

[4] D.R. Diercks, B.P. Gorman, J. Phys. Chem. C, (2015), p. 20623.

[5] J.D. Poplawsky et al., Sol. Energy Mater. Sol. Cells, (2016), 150, p. 95.

[6] Research supported by the US Department of Energy (DOE) Office of Energy Efficiency and Renewable Energy, Foundational Program to Advance Cell Efficiency (F-PACE), grant number DEFOA-0000492, and by ORNL's Center for Nanophase Materials Sciences (CNMS), which is a DOE Office of Science User Facility. J.P. was supported in part by ORNL's laboratory directed research and development (LDRD) program.
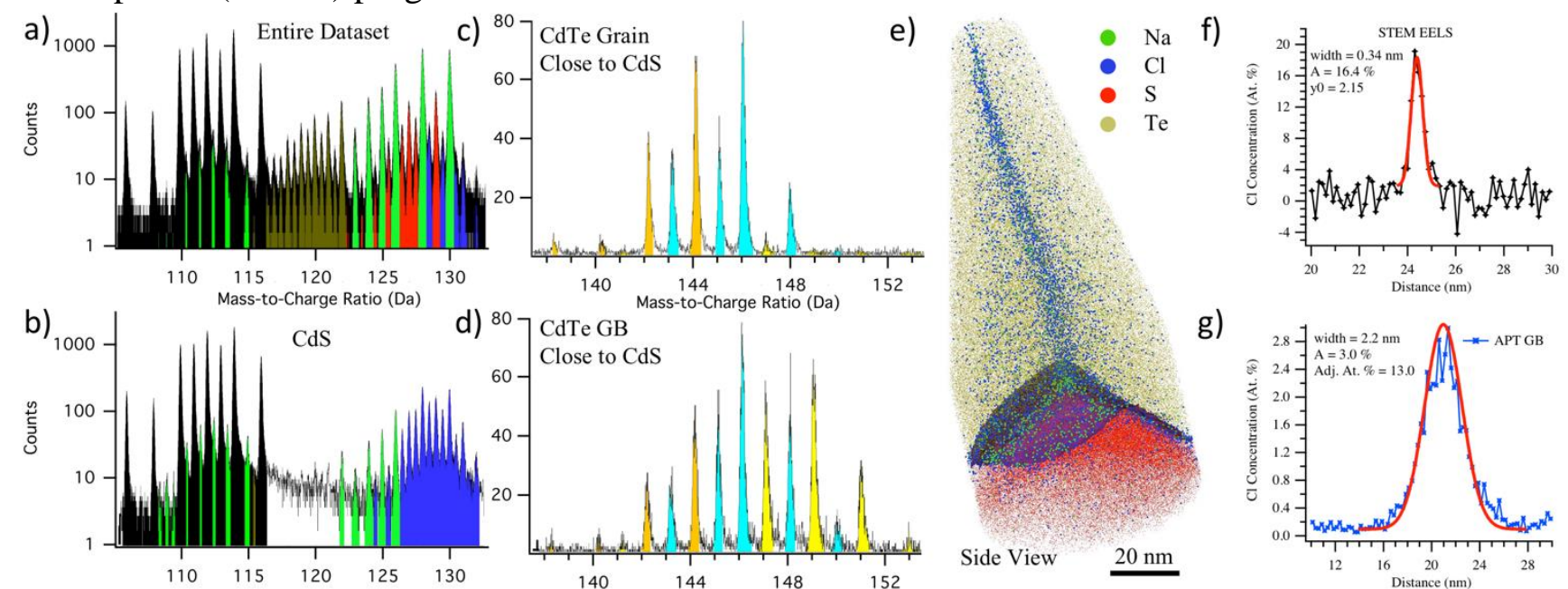

g)

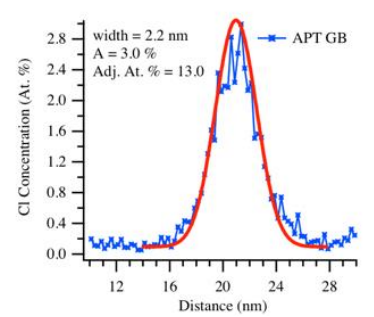

Figure 1. (a)-(d) Mass spectrum of the APT data displayed in (e) with ions ranged as Cd (black), $\mathrm{Cd}_{2}$ (green), CdTe (dark yellow), Te (green), $\mathrm{Te}_{2}$ (red), $\mathrm{Cd}_{2} \mathrm{~S}$ (purple), $\mathrm{CdS}$ (orange), $\mathrm{CdCl}$ (yellow), and major overlaps between $\mathrm{CdS}$ and $\mathrm{CdCl}$ (blue). (f) and (g) STEM EELS and APT Cl profiles, respectively. (e)-(g) are also shown in Ref. [5]. 